S. KIMURA

KODAI MATH. J.

13 (1990), 22-29

\title{
ENTIRE FUNCTIONS WITH RADIALLY DISTRIBUTED ZEROS
}

\author{
By SHIgeru KimurA
}

1. In our previous paper [1], we considered the entire functions of positive integral order and obtained the following characterization of the exponential function.

THEOREM A. Suppose that $f(z)$ is an entire function of positive integral order $p$, and that $f(z)$ has no zeros in a sector $\{z ;|\arg z|<\pi-\pi / 2 p+\eta\} \quad \eta>0)$ and $\delta(0, f)=1$. If there exists a Jordan curve $l$ joining $z=0$ to $z=\infty$ such that

$$
f(z) f(\omega z) \cdots f\left(\omega^{2 p-1} z\right)=O(1) \quad(z \in l)
$$

where $\omega=\exp (\pi i / p)$, then $f(z)=e^{P(i)}$ where $P(z)$ is a polynomial of degree $p$, or else

$$
\lim _{r \rightarrow \infty} \frac{|\log | f(r)||}{r^{p}}=+\infty
$$

In this paper, we show that we can remove the condition on the deficiency. But we confine the distribution of zeros in a sector with half opening and prove the following.

THEOREM 1. Suppose that $f(z)$ is an entire function of positive integral order $p$, and that $f(z)$ has only zeros in a sector $\{z ;|\arg z-\pi| \leqq \pi / 4 p-\eta=\alpha\} \quad(\eta>0)$. If there exists a Jordan curve $l$ joining $z=0$ to $z=\infty$ such that

$$
f(z) f(\omega z) \cdots f\left(\omega^{2 p-1} z\right)=O(1) \quad(z \in l)
$$

where $\omega=\exp (\pi i / p)$, then $f(z)=e^{P(z)}$ where $P(z)$ is a polynomial of degree $p$, or else

$$
\lim _{r \rightarrow \infty} \frac{|\log | f(r)||}{r^{p}}=+\infty
$$

In our previous paper [1], we also considered the entire function of order $q=2 p+1$ having only negative zeros and obtained the following characterization of the exponential function.

Received March 13, 1989 
THEOREM B. Suppose that $f(z)$ is an entire function of order $q=2 p+1$ where $p$ is a non-negative integer, having only negative zeros and $\delta(0, f)=1$. Further setting $\phi\left(z^{2}\right)=f(z) f(-z), g(z)=\phi(-z) / \phi(0)=e^{Q(z)} g_{1}(z)$ where $Q(z)$ is a polynomial and $g_{1}(z)$ is a canonical product, we assume that there is an arbitrarily small $\beta>0$ such that

$$
|\log | g\left(r e^{\imath \beta}\right) g\left(r e^{-\imath \beta}\right)|-2(\cos \beta q / 2) \log | g(r)|| \leqq \varepsilon(r)|\log | g(r)||
$$

for all sufficiently large $r$ where $0 \leqq \varepsilon(r)=O\left(1 / r^{\varepsilon_{0}}\right), \varepsilon_{0}>0$ unless $g(z)$ is in case $\operatorname{deg}(\operatorname{Re} Q(r))=0$ and $g_{1}(r) \equiv 1$. Then $f(z)=e^{P(z)}$ where $P(z)$ is a polynomial of degree $q$, or else

$$
\lim _{r \rightarrow \infty} \frac{-\log |f(r)|}{r^{q}}=+\infty
$$

In our previous paper [2], we considered the entire functions with zeros distributed in a sector. But we obtained only an incomplete result there.

In this paper, we show that we can remove the condition on the deficiency and that the zeros can be distributed in a sector and prove the followings.

LEMMA. Suppose that $g(z)=e^{Q(z)} g_{1}(z)$ is an entire function of finite order having only zeros in a sector $\{z ;|\arg z-\pi| \leqq 2 \alpha<\pi / 2(k+1)\}$, where $Q(z)$ is a polynomial, $g_{1}(z)$ is a canonical product and $k$ is the genus of $g_{1}(z)$. Then the sign of $\log |g(r)|$ is definite for $r \geqq r_{0}$ where $r_{0}$ is a positive number, unless

$$
\operatorname{deg}(\operatorname{Re} Q(r))=0 \text { and } g_{1}(z) \equiv 1 .
$$

TEEOREM 2. Suppose that $f(z)$ is an entire function of order $q=2 p+1$ where $p$ is a non-negative integer, having only zeros in a sector $\{z ;|\arg z-\pi| \leqq \alpha\}$. Further setting $\phi\left(z^{2}\right)=f(z) f(-z), g(z)=\phi(-z) / \phi(0)$, we assume that there exists a positive number $\beta$ such that

$$
\varepsilon \log \left|g\left(r e^{2 \beta}\right) g\left(r e^{-i \beta}\right)\right| \leqq 2 \varepsilon(\cos \beta q / 2) \log |g(r)|+\varepsilon \eta(r) \log |g(r)|,
$$

for all sufficiently large $r$ where $2 \alpha+\beta<\pi /(q+1), \varepsilon= \pm 1, \varepsilon \log |g(r)|>0$ and $0<$ $\eta(r)=O\left(1 / r^{\eta_{0}}\right), \eta_{0}>0$ for all sufficiently large $r$. Then $f(z)=e^{P(z)}$ where $P(z)$ is a polynomial of degree $q$, or else

$$
\lim _{r \rightarrow \infty} \frac{-\log |f(r)|}{r^{q}}=+\infty
$$

Our method of proof depends heavily upon the following formula.

OzAWA Formula [3, p-507]. Let

$$
\phi(x, y)=\frac{1}{2} \log \left(1+2 y \cos x+y^{2}\right)+\sum_{j=1}^{k}(-1)^{\jmath} \frac{y^{\jmath}}{j} \cos j x .
$$


Then

$$
\frac{\partial \phi(x, y)}{\partial x}=\frac{(-1)^{k+1} y^{k+1}}{1+2 y \cos x+y^{2}}(\sin (k+1) x+y \sin k x) .
$$

We remark that $\phi(x, y)=\log \left|E\left(-y e^{\imath x}, k\right)\right|$, where $E$ is the Weierstrass primary factor.

2. Proof of Theorem 1. Let $f(z)$ be an entire function satisfying the hypotheses in Theorem 1 . We can write

$$
f(z)=e^{P(z)} f_{1}(z)
$$

where $P(z)$ is a polynomial of degree at most $p$ and $f_{1}(z)$ is a canonical product with zeros $\left\{a_{\nu}\right\}$. Then we suppose that $f_{1}^{*}(z)$ is a canonical product with zeros $\left\{-\left|a_{\nu}\right|\right\}$. If the genus of $f_{1}(z)$ is $p$, then we have from Ozawa's formula,

$$
\begin{aligned}
& (-1)^{p} \log \left|f_{1}(r)\right| \geqq(-)^{p} \log \left|f_{1}^{*}\left(r e^{\imath \alpha}\right)\right| \\
& =r^{p+1} \int_{0}^{\infty} \frac{n(x)}{x^{p+1}} \frac{x \cos (p+1) \alpha+r \cos p \alpha}{x^{2}+r^{2}+2 x r \cos \alpha} d x \\
& \geqq\left(r^{p+1} \cos (p+1) \alpha\right) \int_{0}^{\infty} \frac{n(x)}{x^{p+1}} \frac{d x}{x+r} \geqq \frac{1}{2}\left(r^{p} \cos (p+1) \alpha\right) \int_{0}^{r} \frac{n(x)}{x^{p+1}} d x,
\end{aligned}
$$

and we have (2). Therefore, by the assumption that (2) is false, we see that the genus of $f_{1}(z)$ is at most $p-1$. Hence we have

Putting

$$
\log M\left(r, f_{1}\right)=o\left(r^{p}\right) .
$$

$$
\phi(\zeta)=\phi\left(z^{2 p}\right)=f_{1}(z) f_{1}(\omega z) \cdots f_{1}\left(\omega^{2 p-1} z\right),
$$

we have $\log M\left(r^{2 p}, \phi\right) \leqq 2 p \log M\left(r, f_{1}\right)=o\left(r^{p}\right)$. Therefore it follows that

$$
\lim _{\rho \rightarrow \infty} \frac{\log M(\rho, \phi)}{\rho^{1 / 2}}=0, \quad\left(\rho=|\zeta|=|z|^{2 p}\right) .
$$

On the other hand, by the assumption (1) we have

$$
m(\rho, \phi) \leqq K<+\infty,
$$

and it follows that $\phi$ satisfies hypothesis in Kjellberg's Lemma $[1, \mathrm{p}-19]$ with $\lambda=1 / 2$ unless $\phi(z)$ is constant. Thus we have

$$
\lim _{\rho \rightarrow \infty} \frac{\log M^{\prime}(\rho, \phi)}{\rho^{1 / 2}}=\beta, \quad 0<\beta \leqq+\infty,
$$

which is a contradiction.

If $\phi$ is constant, then we see that $f(z)=e^{P(z)}$, where $P(z)$ is a polynomial of degree $p$.

3. Proof of Lemma. If $g_{1}(z)$ has zeros $\left\{b_{\nu}\right\}$, then we suppose that $g_{1}^{*}(z)$ is 
a canonical product with zeros $\left\{-\left|b_{\nu}\right|\right\}$. From Ozawa's formula, we have

$$
\begin{aligned}
& (-1)^{k} \log \left|g_{1}(r)\right| \geqq(-1)^{k} \log \left|g_{1}^{*}\left(r e^{2 i \alpha}\right)\right| \\
& =r^{k+1} \int_{0}^{\infty} \frac{n(x)}{x^{k+1}} \frac{x \cos 2 \alpha(k+1)+r \cos 2 \alpha k}{x^{2}+r^{2}+2 r x \cos 2 \alpha} d x \\
& \geqq r^{k+1} \cos 2 \alpha(k+1) \int_{0}^{\infty} \frac{n(x)}{x^{k+1}} \frac{d x}{x+1}>\frac{1}{2} r^{k} \cos 2 \alpha(k+1) \int_{0}^{r} \frac{n(x)}{x^{k+1}} d x .
\end{aligned}
$$

Since $2 \alpha(k+1)<\pi / 2$, it follows that

$$
(-1)^{k} \frac{\log \left|g_{1}(r)\right|}{r^{k}} \geqq \frac{1}{2} \cos 2 \alpha(k+1) \int_{0}^{r} \frac{n(x)}{x^{k+1}} d x \longrightarrow+\infty \quad(r \rightarrow+\infty),
$$

unless case (3). Hence, if $k \geqq l=\operatorname{deg}(\operatorname{Re} Q(r))$, then $\operatorname{sign}\left(\log |g(r)|=\operatorname{sign}\left(\log \left|g_{1}(r)\right|\right)\right.$ and the sign of $\log |g(r)|$ is definite for $r \geqq r_{0}$ where $r_{0}$ is a positive number.

If $k<l$, then we have from Ozawa's formula,

$$
\begin{aligned}
& (-1)^{k} \log \left|g_{1}(r)\right| \leqq(-1)^{k} \log \left|g_{1}^{*}(r)\right|=r^{k+1} \int_{0}^{\infty} \frac{n(x)}{x^{k+1}} \frac{d x}{x+r} \\
& \leqq r^{k} \int_{0}^{r} \frac{n(x)}{x^{k+1}} d x+r^{k+1} \int_{r}^{\infty} \frac{n(x)}{x^{k+2}} d x={ }_{o}(\operatorname{Re} Q(r)) .
\end{aligned}
$$

Hence $\operatorname{sign}(\log |g(r)|)=\operatorname{sign}(\operatorname{Re} Q(r))$ and the sign of $\log |g(r)|$ is definite for $r \geqq r_{0}$ where $r_{0}$ is a positive number.

4. Proof of Theorem 2. Let $f(z)$ be an entire function satisfying the hypotheses in Theorem 2 . We can write

$$
f(z)=e^{P(z)} f_{1}(z)
$$

where $P(z)$ is a polynomial of degree at most $q$ and $f_{1}(z)$ is a canonical product. We suppose that (5) is false. Then, proceeding as in $\S 2$ we see that the genus of $f_{1}(z)$ is at most $q-1=2 p$. Hence we have

$$
\lim _{r \rightarrow \infty} \frac{\log M(r, g)}{r^{q / 2}}=0 .
$$

Now we can write

$$
g(z)=e^{Q(z)} g_{1}(z),
$$

where $Q(z)$ is a polynomial of degree at most $p$ and the genus of the canonical product $g_{1}(z)$ is not greater than $p$.

We can easily deal with case (3). In this case we have

$$
g(z)=\phi(-z) / \phi(0)=\exp \left\{i\left(\alpha_{k^{\prime}} z^{k^{\prime}}+\cdots+\alpha_{1} z\right)\right\},
$$

where $\alpha,\left(j=1, \cdots, k^{\prime}\right)$ are all real. Hence we have $f(z)=\exp (P(z))$ where $P(z)$ 
is a polynomial of degree $q$, which is the desired result.

Now we consider the other cases than (3).

Case (1). $\quad \log |g(r)|>0$ and

$$
\log \left|g\left(r e^{\imath \beta}\right) g\left(r e^{-\imath \beta}\right)\right|-2(\cos \beta q / 2) \log |g(r)| \leqq \eta(r) \log |g(r)|
$$

for all sufficiently large $r$.

We set

and

$$
Q(z)=a_{k^{\prime}} z^{k^{\prime}}+\cdots a_{1} z, \quad \operatorname{deg}(\operatorname{Re} Q(r))=l \quad\left(\leqq k^{\prime}\right)
$$

$$
\arg a_{\jmath}=\theta_{\jmath} \quad\left(j=1, \cdots, k^{\prime}\right) .
$$

We define a harmonic function $H\left(r e^{i \theta}\right)$ in $D=\{z ; 0<|z|<R, 0<\arg z<\beta\}$ as follows,

$$
\begin{aligned}
H\left(r e^{i \theta}\right)= & \int_{-\theta}^{\theta} \log \left|g\left(r e^{\imath \phi}\right)\right| d \phi \\
= & \frac{2}{l}\left|a_{l}\right| r^{l} \sin l \theta \cos \theta_{l}+\cdots+2\left|a_{1}\right| r \sin \theta \cos \theta_{1} \\
& +\int_{-\theta}^{\theta} \log \left|g_{1}\left(r e^{\imath \phi}\right)\right| d \phi .
\end{aligned}
$$

Furthermore we consider the subcases, denoting the genus of $g_{1}(z)$ by $k$.

Case $(1-1) . \quad k \geqq l$. In this case the sign of $\log |g(r)|$ coincides with the one of $\log \left|g_{1}(r)\right|$ for all sufficiently large $r$.

Setting $I_{1}=[0, \pi / 2) \cup(3 \pi / 2,2 \pi], I_{2}=(\pi / 2,3 \pi / 2)$ we define

$$
\begin{aligned}
& H_{1}\left(r e^{i \theta}\right)=\sum_{\theta \in I_{1}} \frac{2}{\jmath}\left|a_{j}\right| r^{\jmath} \sin j \theta \cos \theta_{j}+H_{3}\left(r e^{i \theta}\right), \\
& H_{2}\left(r e^{i \theta}\right)=\sum_{\theta \in I_{2}} \frac{2}{j}\left|a_{j}\right| r^{\jmath} \sin j \theta \cos \theta_{\jmath}
\end{aligned}
$$

where

$$
H_{3}\left(r e^{i \theta}\right)=\int_{-\theta}^{\theta} \log \left|g_{1}\left(r e^{\imath \phi}\right)\right| d \phi=\int_{0}^{\theta} \log \left|g_{1}\left(r e^{\imath \phi}\right) g_{1}\left(r e^{-\imath \phi}\right)\right| d \phi .
$$

If $g_{1}(z)$ has zeros $\left\{b_{\nu}\right\}$, then we have from Ozawa's formula

$$
\begin{aligned}
& \left.\frac{\partial^{2} H_{3}}{\partial \theta^{2}}\right|_{\theta=\beta}=\left.\frac{\partial}{\partial \theta}\left(\log \left|g_{1}\left(r e^{i \theta}\right) g_{1}\left(r e^{-i \theta}\right)\right|\right)\right|_{\theta=\beta} \\
& \quad=\left.\sum_{\nu=1}^{\infty} \frac{\partial}{\partial \theta}\left(\log \left|E\left(\frac{r e^{i \theta}}{b_{\nu}}, k\right) E\left(\frac{r e^{i \theta}}{\bar{b}_{\nu}}, k\right)\right|\right)\right|_{\theta=\beta} \\
& \leqq-(\sin k \beta)^{2} \sum_{\nu=1}^{\infty} \frac{\left(r /\left|b_{\nu}\right|\right)^{k+2}}{\left(r / b_{\nu} \mid+1\right)^{2}} \leqq-\frac{k}{4}(\sin k \beta)^{2} r^{k} \int_{0}^{r} \frac{n(t)}{t^{k+1}} d t .
\end{aligned}
$$

Hence we have 


$$
\left.\frac{\partial^{2} H}{\partial \theta^{2}}\right|_{\theta=\beta}<0,
$$

for all sufficiently large $r$ and therefore we have from the harmonicity of $H\left(r e^{i \theta}\right)$,

$$
\frac{\partial^{2} H}{\partial(\log r)^{2}}=r^{2}\left(\frac{\partial^{2} H}{\partial r^{2}}+\frac{1}{r} \cdot \frac{\partial H}{\partial r}\right)>0
$$

with $\theta=\beta$, for all sufficiently large $r$. From Ozawa's formula again we have

$$
H_{3}\left(r e^{i \beta}\right) \geqq \beta r^{k}(\cos (k+1)(2 \alpha+\beta)) \int_{0}^{r} \frac{n(x)}{x^{k+1}} d x,
$$

and $H\left(r e^{\imath \beta}\right)$ is unbounded. Thus we see that $H\left(r e^{\imath \beta}\right)$ is an increasing convex function of $\log r$ for all sufficiently large $r$.

Proceeding as in $[1, \mathrm{p}-26,27]$, from (4) and (6), we find a sequence of $r=$ $\left\{r_{n}\right\}$ tending to infinity with $n$ such that

$$
C_{1} \frac{\log |g(r)|}{r^{q / 2}}-C_{2} \frac{\log M(2 s, g)}{(2 s)^{q / 2}} \leqq C \frac{\log |g(r)|}{r^{q / 2+\eta_{0}}},
$$

where $C_{1}, C_{2}$ and $C$ are positive constants which do not depend on $r$ and $s$ $(>r)$. For each fixed $r$, if $s$ tends to $\infty$, then we arrive at an impossible inequality from $\eta_{0}>0$.

Case (1-2). $\quad l>k$. In this case, since $R e(Q(r))$ is positive for all sufficiently large $r, \theta_{l}$ lies in $I_{1}=[0, \pi / 2) \cup(3 \pi / 2,2 \pi]$.

Firstly we assume that $k$ is even. In this case, we use the functions $H$, $H_{1}, H_{2}$ and $H_{3}$ defind by (7). $H_{1}\left(r e^{\imath \beta}\right)$ is a nondecreasing convex function of $\log r$ on $(0, \infty)$ and $H_{1}(0)=H_{1}(0+)=0$. Since the degree of $H_{1}\left(r e^{\imath \beta}\right)-H_{3}\left(r e^{\imath \beta}\right)$ is higher than one of $H_{2}\left(r e^{2 \beta}\right), H\left(r e^{2 \beta}\right)$ is a nondecreasing convex function of $\log r$ for all sufficiently large $r$. Hence arguments similar to those in case (1-1) lead to a contradiction.

Secondly we assume that $k$ is odd. In this case we define

$$
\begin{aligned}
& H_{1}\left(r e^{i \theta}\right)=\sum_{\theta \in I_{1}} \frac{2}{j}\left|a_{j}\right| r^{\jmath} \sin j \theta \cos \theta_{\jmath}, \\
& H_{2}\left(r e^{i \theta}\right)=\sum_{\theta \in I_{2}} \frac{2}{j}\left|a_{j}\right| r^{\jmath} \sin j \theta \cos \theta_{j}+H_{3}\left(r e^{i \theta}\right),
\end{aligned}
$$

where

$$
H_{3}\left(r e^{i \theta}\right)=\int_{0}^{\theta} \log \left|g_{1}\left(r e^{\imath \phi}\right) g_{1}\left(r e^{-\imath \phi}\right)\right| d \phi .
$$

Then we have $H\left(r e^{i \theta}\right)=H_{1}\left(r e^{i \theta}\right)+H_{2}\left(r e^{i \theta}\right)$.

It is trivial that $H_{1}\left(r e^{i \beta}\right)$ is a nondecreasing convex function of $\log r$ on $(0, \infty)$ with $H_{1}(0)=H_{1}(0+)=0$.

Now we show that $H\left(r e^{i \beta}\right)$ is a nondecreasing convex function of $\log r$ for all sufficiently large $r$. 
We have from Ozawa's formula

$$
\begin{aligned}
-H_{3}\left(r e^{2 \beta}\right) & \leqq 2 \beta r^{k+1} \int_{0}^{\infty} \frac{n(x)}{x^{k+1}} \frac{d x}{x+r} \\
& \leqq 2 \beta r^{k} \int_{0}^{r} \frac{n(x)}{x^{k+1}} d x+2 \beta r^{k+1} \int_{r}^{\infty} \frac{n(x)}{x^{k+2}} d x=o\left(r^{l}\right) \quad(r \rightarrow \infty) .
\end{aligned}
$$

Hence $\left|H_{3}\left(r e^{\imath \beta}\right)\right| / r^{l} \rightarrow 0$ as $r \rightarrow+\infty$ and $H\left(r e^{\imath \beta}\right)$ is unbounded.

If $g_{1}(z)$ has zeros $\left\{b_{\nu}\right\}$, then we have from Ozawa's formula again,

$$
\begin{aligned}
\left.\frac{\partial^{2} H_{3}}{\partial \theta^{2}}\right|_{\theta=\beta} & \leqq \frac{2}{\cos (\beta+2 \alpha)} \sum_{\nu=1}^{\infty} \frac{\left(r /\left|b_{\nu}\right|\right)^{k+1}}{\left(1+r /\left|b_{\nu}\right|\right)^{2}}\left\{1+\frac{r}{\left|b_{\nu}\right|}\right\} \\
& \leqq \frac{2(k+1) r^{k+1}}{\cos (\beta+2 \alpha)} \int_{0}^{\infty} \frac{n(t)}{t^{k+1}} \frac{d t}{1+r / t} \\
& \leqq \frac{2(k+1) r^{k+1}}{\cos (\beta+2 \alpha)} \frac{1}{r} \int_{0}^{r} \frac{n(t)}{t^{k+1}} d t+\frac{2(k+1) r^{k+1}}{\cos (\beta+2 \alpha)} \int_{r}^{\infty} \frac{n(t)}{t^{k+2}} d t .
\end{aligned}
$$

Hence $\left(\partial^{2} H / \partial \theta^{2}\right)_{\theta=\beta}$ is negative and $\left(\partial^{2} H / \partial(\log r)^{2}\right)_{\theta=\beta}$ is positive for all sufficiently large $r$. Therefore $H\left(r e^{2 \beta}\right)$ is a nondecreasing convex function of $\log r$ for all sufficiently large $r$. Thus arguments similar to those in case (1-1) lead to a contradiction.

Case (2). $\quad \log |g(r)|<0 \quad$ and $\quad \log \left|g\left(r e^{\imath \beta}\right) g\left(r e^{-\imath \beta}\right)\right|-2(\cos \beta q / 2) \log |g(r)| \geqq$ $\eta(r) \log |g(r)|$ for all sufficiently large $r$.

Put $\widetilde{Q}(z)=-Q(z), \quad \tilde{g}_{1}(z)=g_{1}(z)^{-1}$ and $\tilde{g}(z)=e^{\tilde{Q}(z)} \tilde{g}_{1}(z)$. Then (4) is equivalent to

$$
\log \left|\tilde{g}\left(r e^{\imath \beta}\right) \tilde{g}\left(r e^{-\imath \beta}\right)\right|-2(\cos \beta q / 2) \log |\tilde{g}(r)| \leqq \eta(r) \log |\tilde{g}(r)| .
$$

Thus our case is handled in a fashion almost similar to case (1).

We only show how to handle the inequality corresponding to (8). Proceeding as in case (1-1), we have

$$
C_{1} \frac{\log |\tilde{g}(r)|}{r^{q / 2}}-C_{2} \frac{\log M_{\beta}(2 s, \tilde{g})}{(2 s)^{q / 2}} \leqq C \frac{\log |\tilde{g}(r)|}{r^{q / 2+\eta_{0}}},
$$

where $M_{\beta}(2 s, \tilde{g})=\sup _{|\theta|<\beta}\left|\tilde{g}\left(2 s e^{i \theta}\right)\right|$. In this inequality we must show that

$$
\lim _{r \rightarrow \infty} \frac{\log M_{\beta}(r, \tilde{g})}{r^{q / 2}}=0 .
$$

Since $\log M_{\beta}(r, \tilde{g}) \leqq \sup _{\mid \theta_{\mid<\beta}} \operatorname{Re}\left(\tilde{Q}\left(r e^{i \theta}\right)\right)+\log M_{\beta}\left(r, \tilde{g}_{1}\right)$ and $\lim _{r \rightarrow \infty}\left\{\sup _{\mid \theta_{1}<\beta} \operatorname{Re}\left(R\left(\tilde{Q}\left(r e^{i \theta}\right)\right)\right\} / r^{q / 2}\right.$ $=0$, it is sufficient to show that

$$
\lim _{r \rightarrow \infty} \frac{\log M_{\beta}\left(r, \tilde{g}_{1}\right)}{r^{q / 2}}=0
$$


in the case that the genus of $g_{1}(z)$ is not smaller than the degree of $\operatorname{Re}(Q(r))$.

Since (6) implies $\lim _{r \rightarrow \infty}\left\{\log M\left(r, g_{1}\right)\right\} / r^{q / 2}=0$, we have $m_{\beta}\left(r, \tilde{g}_{1}\right) / r^{q / 2} \rightarrow 0$ as $r \rightarrow \infty$, where

$$
m_{\beta}\left(r, \tilde{g}_{1}\right)=\frac{1}{2 \pi} \int_{-\beta}^{\beta} \log ^{+}\left|\tilde{g}_{1}\left(r e^{i \theta}\right)\right| d \theta=\frac{1}{2 \pi} \int_{-\beta}^{\beta}\left(-\log \left|g_{1}\left(r e^{i \theta}\right)\right|\right)^{+} d \theta .
$$

Now in this case, we have from Ozawa's formula for $\theta(|\theta| \leqq \beta)$,

$$
\begin{aligned}
-\log \left|g_{1}\left(r e^{i \theta}\right)\right| & \geqq r^{k+1} \int_{0}^{\infty} \frac{n(x)}{x^{k+1}} \frac{x \cos (k+1)(\beta+2 \alpha)+r \cos k(\beta+2 \alpha)}{x^{2}+r^{2}+2 x r \cos (\beta+2 \alpha)} d x \\
& \geqq(\cos (k+1)(\beta+2 \alpha)) r^{k+1} \int_{0}^{\infty} \frac{n(x)}{x^{k+1}} \frac{d x}{x+r} \\
& \geqq(\cos (k+1)(\beta+2 \alpha)) \log M_{\beta}\left(r, \tilde{g}_{1}\right) .
\end{aligned}
$$

Hence we obtain (10).

Proceeding as in case (1), we have a contradiction from (9).

\section{REFERENCES}

[1] S. KimURA, A characterization of the exponential function by product, Kodai Math. J., 7 (1984), 16-33.

[2] S. KIMURA, A characterization of the exponential function and Lindelöf function, Kodai Math. J., 9 (1986), 351-360.

[3] M. OzAwA, Radial distribution of zeros and deficiency of a canonical product of finite genus, Kodai Math. Sem. Rep. 24 (1972), 502-512.

Department of Mathematics,

UTSUNOMIYA UNIVERSITY

Mine-Machi, Utsunomiya, Japan 